\title{
Reaction Systems: A Formal Framework for Processes
}

\author{
Grzegorz Rozenberg \\ Leiden University, Leiden Centre of Advanced Computer Science, Niels Bohrweg 1, \\ 2333 CA Leiden, The Netherlands
}

\begin{abstract}
The functioning of a living cell consists of a huge number of individual reactions that interact with each other. These reactions are regulated, and the two main regulation mechanisms are facilitation/acceleration and inhibition/retardation. The interaction between individual biochemical reactions takes place through their influence on each other, and this influence happens through the two mechanisms mentioned above.

In our lecture we present a formal framework for the investigation of biochemical reactions - it is based on reaction systems. We motivate this framework by explicitely stating a number of assumptions/axioms that (we believe) hold for a great number of biochemical reactions - we point out that these assumptions are very different from the ones underlying traditional models of computation such as Petri Nets. We discuss some basic properties of reaction systems, and demonstrate how to capture and analyze, in our formal framework, some biochemistry related notions.

The lecture is of a tutorial character and self-contained.
\end{abstract}

